\title{
A DENIS SURVEY OF STAR FORMING REGIONS
}

\author{
E. COPET
}

Observatoire de Paris

\section{Introduction}

A complete census of embedded stellar population can be made by exploring in the infrared large areas of the sky in which giant molecular clouds extend. Very recently, thanks to the of large format IR array detectors, studies of young stellar population in GMCs, have been undertaken by different authors (i.e., Lada et al. 1991) but all these observations were limited to relatively small regions of the whole GMCs, the DENIS project (Epchtein, this volume, p. 106) surveys the south hemisphere at $\mathrm{I}, \mathrm{J}$ and $\mathrm{K}_{s}$ bands, including most of these clouds.

\section{Orion A}

The Orion molecular cloud complex is the best studied GMC of our Galaxy, given its short distance ( $\mathrm{D}=450 \mathrm{pc} ;$ e.g., Genzel \& Stutzki 1989). During the proto-survey period, the DENIS instrument has observed this wellknown cloud at $\mathrm{J}$ and $\mathrm{K}_{s}$ band.

\subsection{OBSERVATIONS AND RESULTS}

The observations of the region were made in february 1995 using the DENIS standard procedure (Epchtein et al. 1994). For the purpose of this study, 6 "DENIS strips" were used to cover the 3 square degrees around the Trapezium. The data reduction and the source extraction method is presented in more details in Copet et al. (in preparation).

We estimate the completeness limit of our sample at 15.6 and 13.8 at $\mathrm{J}$ and $\mathrm{K}_{s}$ band, respectively. The number of sources extracted above the completeness limit are 7032 at $\mathrm{K}_{s}$ and 7621 at $\mathrm{J}$. The spatial distribution of the stars detected at the $\mathrm{K}_{s}$ band is presented in Figure 1. The $\mathrm{J}$ and $\mathrm{K}_{s}$ 


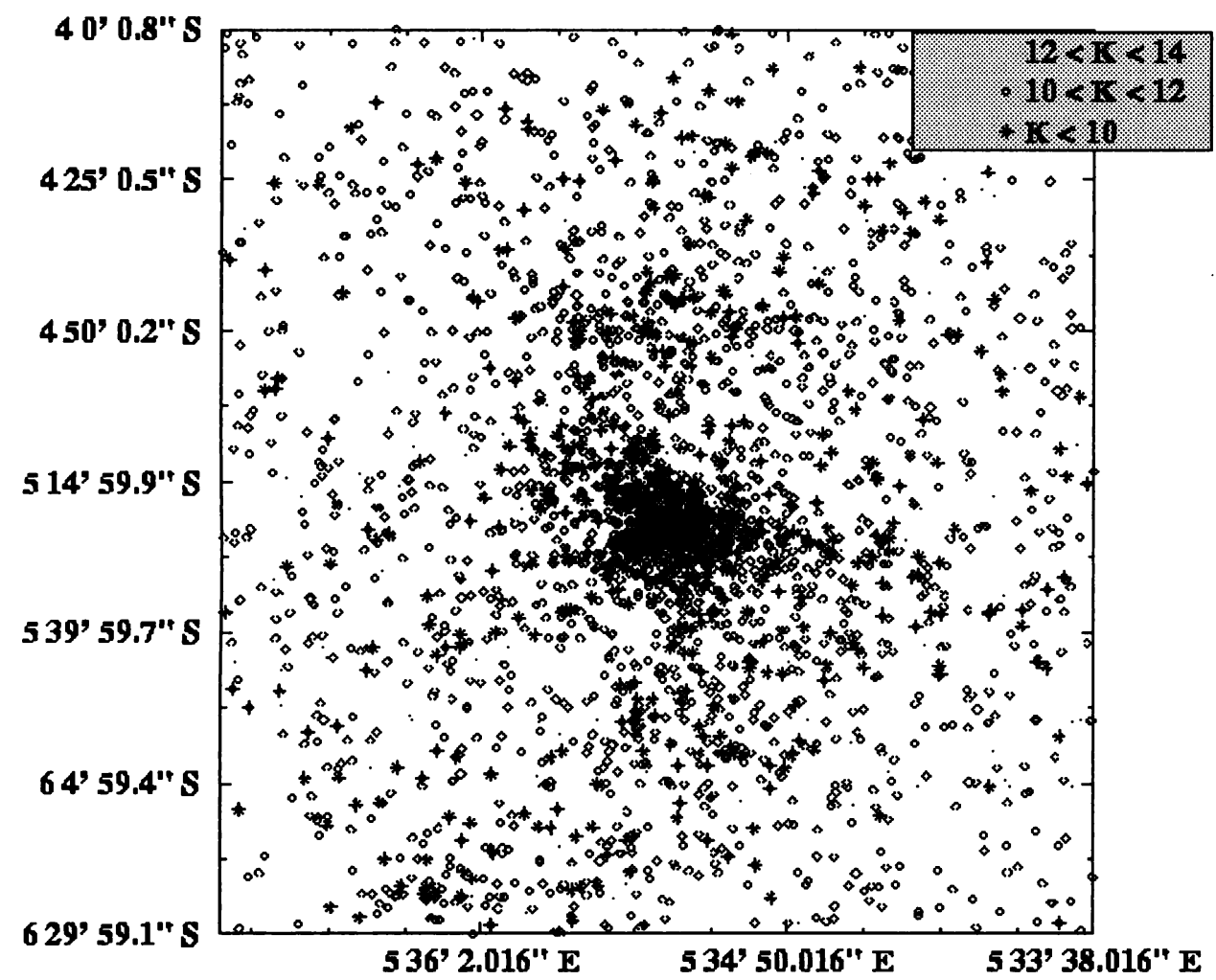

Figure 1. Spatial distribution of the sources detected at $\mathrm{K}_{s}$ band according 3 ranges of magnitude. The equinox of the coordinates is 2000.0

luminosity functions (not shown) present a clear excess of high luminosity stars in the vicinity of the Trapezium. The $(\mathrm{J}-\mathrm{K})$ color of the stars follows the $\mathrm{CO}$ density distribution, but some very red objects $(\mathrm{J}-\mathrm{K}>3)$ could be found far away the $\mathrm{CO}$ density peaks.

Using a molecular CS survey of this region (Tatematsu et al. 1993) and correlating our catalog with the CS peaks location, we have detected inside the CS cores 114 sources at $\mathrm{J}$ and 906 sources, in the $\mathrm{K}_{s}$ band. Probably as only $10 \%$ of these sources are associated with the cores, then, usually rich clusters of embedded objects are not observed.

\section{Chamaeleon}

The Chamaeleon region has been mapped at several wavelengths: IR (Gauvin et al. 1992), X ( Huenemoerder et al. 1994). The DENIS project has 

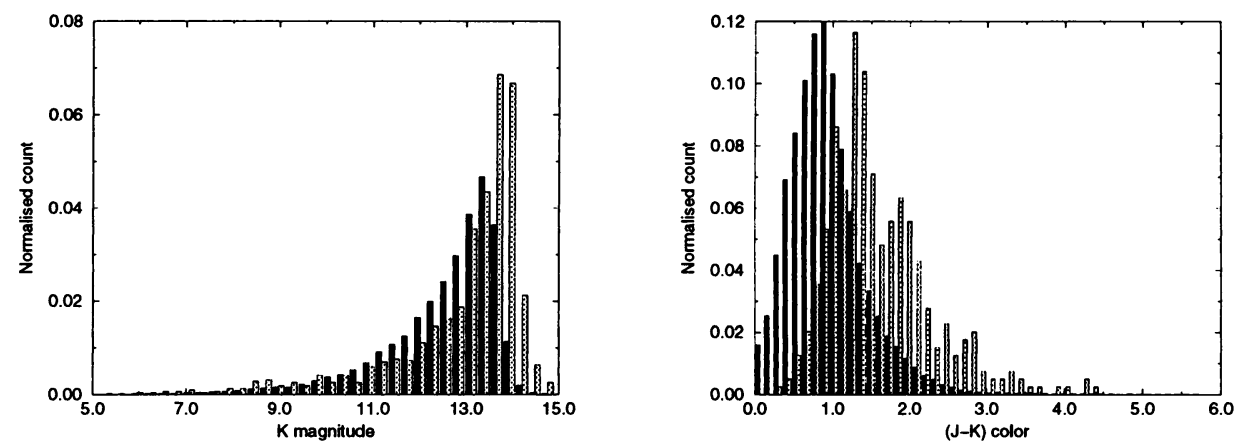

Figure 2. Histograms obtained with the DENIS data in the Chamaeleon region. The left side presents the $\mathrm{K}$ luminosity function in the Cha I region (grey bars) while the right side represents the distribution of the $(\mathrm{J}-\mathrm{K})$ color in the same area. The black bars are the distribution derived from a 30 degree long strip for comparison. The counts are normalised to unity.

already surveyed a large area on the Cha I and Cha II clouds in the $\mathrm{J}$ and $\mathrm{K}_{s}$ bands. We present in Figure 2 the luminosity function and the color histogram for the Cha I region. The number of $\mathrm{K}_{s}$ sources detected in this region is around 3000 .

We note a very faint stellar population in the Cha I cloud $(K>14)$ close to our detection limit and an excess of reddenning, but with only one color, we cannot disentangle between an intrinsic reddenning of the star or an extinction due to the cloud material.

\section{Conclusion}

The preliminary results on these two star-forming regions show that the nominal performances of the instrument are reached. The comparison with ISOCAM data (Kaas A.A., private communication; Nordh et al. 1996) shows a good correlation between the stellar population detected in the Near and Far infrared bands and provide an excellent base to study these kind of regions at higher sensitivity.

\section{References}

Epchtein N., et al., 1994 Astrophys.Space.Sc. 217, 3

Genzel R., Stutzki J., 1989 Ann.Rev.Astron.Astrophys. 27, 41

Lada C.J., DePoy D.L., Merrill K.M., Gatley I., 1991 Astrophys.J. 374, 533

Huenemoerder D.P., Lawson W.A., Feigelson E.D., 1994 Mon.Not.R.astron.Soc. 271, 967

Nordh L., Olofsson G. et al., 1996 Astron.Astrophys. in press

Tatematsu et al., 1993 Astrophys.J. 404, 643 